\title{
Las Rickettsias del grupo de las fiebres manchadas: Respuesta inmune y sus proteínas inmunodominantes
}

\author{
Jorge Zavala $\mathbf{C}^{1}$, Alfredo Ruiz $\mathbf{S}^{2}$, Jorge Zavala $\mathbf{V}^{\mathbf{2}}$. \\ Spotted fever Rickettsiae. Their \\ immunodominant proteins and \\ immune response
}

The organisms of Rickettsia species are Gram (-) bacteria that cause severe illnesses in humans and are an important health problem in several countries around the world, including Mexico. The presence of different antigens between species and also in different strains of the same specie is an obstacle for vaccine development and serological diagnosis. There are important advances in the knowledge of the rickettsial antigenic structure and the resulting immune response in infected hosts, including humans. This review covers these topics and provides an overview about the development of vaccines and accessible diagnostic methods for diseases caused by Rickettsia (Rev Méd Chile 2004; 132: 381-7).

(Key Words: Fever; Immunity, natural; Rickettsia)

Recibido el 29 de julio, 2003. Aceptado en versión corregida el 18 de diciembre, 2003.

${ }^{1}$ Centro de Investigaciones Regionales «D. Hideyo Noguchi»y ${ }^{2}$ Facultad de Medicina, Universidad Autónoma de Yucatán. Mérida Yucatán, México.

L as bacterias del género Rickettsia son responsables de un gran número de enfermedades de importancia, tal como la fiebre manchada de las Montañas Rocosas, la fiebre manchada del Mediterráneo y tifus, entre otras. Estos microorganismos poseen ciclos epidemiológicos complicados y causan cuadros febriles agudos, los cuales a menudo son autolimitados, pero que pueden presentar una secuela a largo plazo o incluso ser fatales, si no hay tratamiento. Las

Correspondencia a: Jorge Zavala $\mathrm{C}, \mathrm{PhD}$, Centro de Investigaciones Regionales «Dr. Hideyo Noguchi», Universidad Autónoma de Yucatán. Av. Itzaes № 490 x 59, CP 97000, Mérida Yucatán, México. Fax (52 999) 923.61.20. E-mail zcastro@tunku.uady.mx especies de la familia Rickettsiaceae son intracelulares obligados, capaces de reproducirse en el núcleo y en el citoplasma de la célula hospedera infectada ${ }^{1}$. El género Rickettsia se divide en tres biotipos, basándose en la respuesta inmunológica y en su organismo vector: El grupo de las fiebres manchadas, el grupo tifo y el tifo de los matorrales; sin embargo, éste último posee una sola especie: $\mathrm{R}$ tsutsugamushi, y ha sido reclasificada en un nuevo género: Orientia ${ }^{2}$.

Las rickettsias son capaces de multiplicarse en una o más especies de artrópodos, y se considera que son huéspedes accidentales en varias especies de mamíferos, incluyendo al humano ${ }^{3,4}$. Las infecciones por rickettsia, inicialmente presentan síntomas sistémicos no específicos como son 
fiebre, dolor de cabeza, mialgia y malestar, lo que hace difícil cualquier diagnóstico temprano basado en una semiología clínica ${ }^{5}$. Signos adicionales, como exantema y la formación de escaras en el sitio de inóculo, pueden ayudar a distinguir esta patología de otras enfermedades febriles, pero pueden no ser definitivos. Trabajos recientes en el Estado de Yucatán, México, demostraron la presencia de $\mathrm{R}$ felis en pulgas (Ctenocephalides felis) y no en garrapatas (Amblyomma cajennense o Rhipicephalus sanguineus), proponiéndose como los vectores usuales de rickettsias del grupo de las fiebres manchadas en México ${ }^{6-8}$.

Antigénicamente, el género Rickettsiae comprende a dos grupos definidos: el grupo de las fiebres manchadas y el grupo tifo. Ambos comparten muchas particularidades, incluyendo una proteína conservada de membrana externa de 135 $\mathrm{kDa}$ designada como rOmpB y una lipoproteína de $17 \mathrm{kDa}$. Adicionalmente, las especies del grupo de las fiebres manchadas comparten entre sí una proteína de membrana externa de 170 a $198 \mathrm{kDa}$ designada como rOmpA ${ }^{9}$. Las rickettsias que producen las fiebres manchadas son transmitidas por la mordedura de garrapatas, pulgas o ácaros. El patógeno invade y prolifera en las células endoteliales (ECs) de vasos pequeños, produciendo focos de destrucción ${ }^{10}$. El daño vascular produce un incremento en la permeabilidad vascular, edema, hipotensión e hipovolemia ${ }^{4}$, y se ha observado que las células endoteliales incrementan significativamente sus niveles de superóxido.

Algunas complicaciones pueden involucrar a distintos órganos, incluyendo al sistema nervioso, y puede causar la muerte, especialmente en aquellos pacientes de edad avanzada, débiles 0 inmunodeprimidos ${ }^{11}$.

Cada vez son más los países latinoamericanos donde se encuentran casos humanos de rickettsiosis, lo que nos hace pensar que se trata de una enfermedad que no se había tomado en cuenta en los diagnósticos diferenciales de las enfermedades febriles que aquejan a la población en general. Con la aparición de nuevos y más sensibles métodos de diagnóstico, los trabajos recientes demuestran un alto porcentaje de positividad hacia las rickettsias entre la población estudiada, lo que puede estar indicando que se trata de un problema de salud más importante de lo esperado.
Las diferencias antigénicas que se han encontrado entre las diferentes especies y cepas de rickettsias, y la falta de conocimiento pleno de la respuesta inmune que provocan en los pacientes, en especial de R felis, han sido un factor importante para detener el desarrollo de vacunas eficaces en el control de la enfermedad. Este trabajo pretende establecer el panorama actual de un aspecto importante de las rickettsiosis como son la respuesta inmune del huésped y sus proteínas inmunodominantes importantes en el desarrollo de vacunas y métodos de diagnóstico específicos.

\section{RESPUESTA INMUNE}

Aunque el mecanismo de defensa del hospedero no es del todo conocido, algunos estudios han señalado que la respuesta inmune celular tiene un papel importante tanto en modelos experimentales como en la infección humana ${ }^{12,13}$. Se considera que la respuesta por anticuerpos puede no ser crucial en el proceso de curación, ya que ensayos en ratones atímicos, se puede lograr una infección exitosa a pesar de una respuesta humoral rigurosa ${ }^{14}$.

En la respuesta inmune celular en pacientes con rickettsiosis, podemos encontrar una clara modificación en las células mononucleares periféricas con una reducción en las células $\mathrm{T}$ circulantes, en particular $\mathrm{CD} 4^{+}$, y $\mathrm{CD} 45^{+15}$. Estas modificaciones pueden estar relacionadas con la adhesión celular al endotelio vascular, seguido por su internalización a los sitios de inflamación ${ }^{16}$. En la fase aguda se observa una ligera disminución de los otros subgrupos celulares, como $\mathrm{T}$ $\mathrm{CD}^{+}$, NK CD16 ${ }^{+}, \mathrm{B} \mathrm{CD} 20^{+}$y un aumento significativo de los monocitos CD $14^{+} / \mathrm{HLA}^{-D R^{+}}$. Todos los subgrupos celulares regresan a sus niveles normales posterior a un tratamiento exitoso, excepto para los monocitos clase CD $14^{+} / \mathrm{HLA}^{-\mathrm{DR}^{+}}$ que persisten con un nivel elevado después de la recuperación del paciente, aparentemente de manera directa con los altos niveles del factor de necrosis tumoral alfa $(\mathrm{TNF} \alpha)^{15}$.

Las líneas celulares de endotelio pueden procesar y presentar exitosamente los antígenos rickettsiales a los linfocitos T CD8 y activarlos; esta activación es determinada por la secreción de interferón gamma (IFN- $\gamma$ ), y ambos son factores 
cruciales para la eliminación de rickettsias y el posterior restablecimiento del paciente ${ }^{17,18}$.

La respuesta inmune humoral del paciente se altera profundamente durante la enfermedad. En la fase aguda, el IFN- $\gamma$, la interleucina-10 (IL-10), la IL-6 y el TNF $\alpha$ se incrementan significativamente en suero en comparación con los controles sanos. Posteriormente, durante la segunda semana después de la infección, los niveles de IFN- $\gamma$ disminuyen con rapidez, en tanto que los valores de IL-6, IL-10 y TNF $\alpha$ declinan gradualmente, hasta llegar a valores dentro del rango de lo normal en la etapa de convalecencia ${ }^{15}$.

La IL-12, que contribuye fuertemente a la resistencia de patógenos, no ha sido detectada en el suero de pacientes en fase aguda. Esta condición se ha explicado porque la detección de IL-12 se presentaría muy tempranamente y por un corto período en respuesta a agentes infecciosos, por lo que la generalidad de los métodos de medición de IL-12 realizados después del cuadro febril puede no ser eficaz ${ }^{19}$. Podemos considerar que una concentración elevada de IL-12 en el suero al día 5 , se relaciona con una respuesta inmune efectiva y específica en pacientes infectados ${ }^{18}$.

El TNF $\alpha$ parece ser importante en el proceso de curación, siendo considerado como un marcador certero para una completa resolución de la infección. Así mismo, se ha mencionado que la activación conjunta de IFN- $\gamma$ y TNF $\alpha$ activa las células endoteliales y a otras células blanco para destruir a organismos intracelulares por un mecanismo dependiente de la síntesis de óxido nítrico, siendo hipotéticamente las fuentes de ambas citocinas, los linfocitos $\mathrm{T}$ y los macrófagos infiltrados ${ }^{20,21}$. Algunos cambios necróticos de las células endoteliales infectadas de $\mathrm{R}$ rickettsii correlacionan con una acumulación dramática de peróxidos intracelulares, incluyendo peróxidos lipídicos y peróxido de hidrógeno ${ }^{22}$.

El IFN- $\gamma$ parece tener un papel importante en la actividad anti-rickettsial durante la fase temprana de la infección. Posteriormente, es relevado por el efecto de IL-10, que actúa en la regulación de los mediadores inflamatorios, y en conjunto con IL-6 activando la respuesta de las inmunoglobulinas en contra de las rickettsias. La otra citocina de respuesta temprana IL- $1 \alpha$ no es secretada al torrente sanguíneo en ninguna fase de la infec- ción, sino que se asocia a las células y contribuye a las respuestas pro coagulante e inflamatoria localizadas, las cuales ocurren durante el curso de la enfermedad ${ }^{23}$.

La fuente principal de protección contra las rickettsias la proveen los linfocitos T CD 8 al participar en la activación de los linfocitos citotóxicos (CTL), los cuales constituyen el mecanismo potencial de eliminación de células infectadas por rickettsias, como lo evidencia la infección con $\mathrm{R}$ conorii de ratones que carecen de linfocitos $\mathrm{T}$ CD8 ${ }^{20}$. Si el efecto de los CTL sobre las células infectadas es la inducción de apoptosis, los cuerpos apoptóticos con un contenido de rickettsias podrían hipotéticamente ser fagocitados rápidamente por células adyacentes (ej: macrófagos perivasculares) y degradados en sus fagolisosomas.

La vacuna de ADN más prometedora en la actualidad utiliza una parte de rOmpA de $\mathrm{R}$ rickettsii que estimula una respuesta inmune protectora hacia una dosis letal de $\mathrm{R}$ conorii en ratones. La producción de IFN- $\gamma$ por los linfocitos $\mathrm{T}$ expuestos a antígenos expresados por las vacunas de ADN, ha indicado una fehaciente estimulación de la respuesta inmune celular ${ }^{24}$.

\section{PROTEÍNAS INMUNODOMINANTES}

Tradicionalmente, la caracterización y clasificación de las especies rickettsiales se ha basado en la tipificación de proteínas antigénicas de superficie celular y las lipoproteínas de la pared celular. Algunas comunicaciones han indicado que los aislados de rickettsia, contienen polipéptidos inmunodominantes que pueden ser tanto antigénicamente idénticos como diferentes entre especies del género Rickettsia ${ }^{1,25}$. Tanto las rickettsias del grupo de las fiebres manchadas como del grupo tifo son genéticamente similares. En los ensayos por Western blot ha sido difícil definir qué bandas representan los antígenos especie específicos debido a la reactividad cruzada existente entre los polipéptidos del grupo de las fiebres manchadas. Este género contiene dos proteínas de membrana: las proteínas rickettsiales externas de membrana $\mathrm{A}$ y B (rOmpA y $\mathrm{rmpB})^{26}$. 
Los métodos de diagnóstico de las enfermedades rickettsiales en general, se han enfocado en la identificación de la lipoproteína de $17 \mathrm{kDa}$ y de rOmpB, por técnicas inmunológicas (IFI, Westem blot) y moleculares (reacción en cadena de la polimerasa (PCR)). En el caso de las rickettsias del grupo de las fiebres manchadas, la caractenzación de especie se realiza por PCR y fragmentos de restricción polimórficos (RFLPs) de la proteína rOmpA.

El gen que expresa rOmpA ha sido identificado en casi todos los miembros del grupo de las fiebres manchadas. El análisis de la secuencia nucleotídica del gen rOmpA sugiere que es una proteína transmembranal, que consiste de varias regiones hidrofílicas que son externas a la bacteria $^{27}$. Presenta un peso molecular de 170 a 198 $\mathrm{kDa}$, y una correlación genética que ha sido estimada revelando unidades de 72-75 aminoácidos codificados por una región repetida en tan$\operatorname{dem}^{28}$. Esta variabilidad genética entre especies de rickettsias ha permitido su empleo como una herramienta útil en la caracterización molecular intra e inter especies ${ }^{29}$.

La utilidad del diagnóstico por Western blot, en las rickettsiosis del grupo de las fiebres manchadas con la proteína rOmpA es limitado, ya que los epítopes son interrumpidos por cambios conformacionales en las moléculas inducidos durante el tratamiento de las muestras con dodecil sulfato de sodio (SDS) y ß-mercaptoetanol previo a la electroforesis (SDS-PAGE) ${ }^{30}$. Sin embargo, es una herramienta útil al comparar con pruebas de inmunofluorescencia indirecta; por ejemplo, la reactividad entre algunos anticuerpos monoclonales para rOmpA contra antígenos purificados de $\mathrm{R}$ rhipicephali, $\mathrm{R}$ rickettsii y las cepas $\mathrm{M}-1$ y Moroccan de R conorii, no ha podido ser demostrada por Western blot, aunque una reactividad positiva fue evidente en las pruebas por IFI. El análisis antigénico y nucleotídico realizado en siete cepas diferentes de $\mathrm{R}$ conorii, ha demostrado que no todos los epítopes de la proteína son expresados en una sola cepa, condición que puede deberse a deleciones significativas que han producido la falta de expresión antigénica del gen rOmpA, o a modificaciones en el marco de lectura abierto con codones de paro prematuro, como se ha descrito en $\mathrm{R}$ felis ${ }^{31,32}$. El diagnóstico certero, y la caracterización específica de especie, se logra con la prueba de PCR de rOmpA complementada con una prueba de RFLPs del fragmento amplificado; la combinación de estas pruebas es particularmente importante para $\mathrm{R}$ felis ${ }^{8}$.

El gen de rOmpA de $\mathrm{R}$ felis codifica para una proteína de menor tamaño $(60.5 \mathrm{kDa})$ y con un contenido de aminoácidos diferente a las regiones repetidas observadas en la proteína rOmpA de otras especies $9,28,32$, esto podría ser indicativo de la posible extinción de un gen de virulencia dentro del género Rickettsia.

Esta «extinción» de genes es uno de los aspectos controversiales de la secuencia genómica de $\mathrm{R}$ prowazekii debido a su bajo contenido de regiones codificantes. La célula hospedera eucariota constituye un medio de crecimiento inusualmente rico en comparación a otros nichos bacterianos. De esta manera, podría esperarse que algunos genes que codifican para proteínas que pueden ser proporcionadas por el citoplasma hospedero, pudieran haberse perdido ${ }^{33}$. De hecho, la mayor parte de los genes que codifican para proteínas involucradas en la biosíntesis de aminoácidos y nucleótidos están ausentes del genoma de $\mathrm{R}$ prowazekii, mientras que la mayor parte de los genes que codifican para proteínas involucradas en funciones de replicación, transcripción y traducción aún están presentes ${ }^{34}$. Parece razonable asumir que las pérdidas masivas de información genética de Rickettsia han dejado trazas en la secuencia nucleotídica del genoma actual, particularmente si los genes están aún siendo modificados o eliminados paso a paso.

La proteína $\mathrm{rOmpB}$ tiene un peso molecular de $135 \mathrm{kDa}$, aunque siempre se ha referido a ella como una proteína de $120 \mathrm{kDa}$ debido a su movilidad electroforética bajo la conformación nodesnaturalizada. Esta proteína de membrana es un componente de la capa superficial de la membrana, y a pesar de que el gen rOmpB pudiera codificar para una proteína de $168 \mathrm{kDa}$, existe evidencia que la proteína es modificada hasta generar una proteína de menor tamaño $(135 \mathrm{kDa})$, observada por electroforesis ${ }^{35}$. En contraste con el grupo de las fiebres manchadas, el grupo tifo expresa $\mathrm{rmpB}$ pero no rOmpA.

Dentro del grupo tifo, rOmpB es también conocida como proteína antigénica de superficie (SPA) y es utilizada para el diagnóstico serológico y por PCR. 
También se utiliza para la caracterización de especie, porque contiene dominios que son responsables para las reacciones serológicas que distinguen a $\mathrm{R}$ typhi y R prowazekii ${ }^{36}$. Se han comunicado mapas de epítopes lineales y algunos sitios de unión para 18 anticuerpos monoclonales que reconocen epítopes no conformacionales y 8 de estos anticuerpos reaccionaron predominantemente con una sola región de las SPAs, correspondiente al tercio amino terminal (aminoácidos 6-211) de R prowazekii36,37. A pesar de que estos anticuerpos presentan una baja reacción cruzada con la SPA intacta de Rtyphi, se ha podido observar que al menos un anticuerpo presenta una reacción detectable con el fragmento T4b, sugiriendo que existen homologías únicamente en este epítope. El análisis general de la secuencia de $\mathrm{rOmpB}$ indica que es un gen bien conservado, especialmente en la región del péptido beta, condición que sugiere que la región amino terminal de las proteínas SPA tienen estructuras secundarias y posiblemente terciarias similares.

Los esfuerzos para la generación de vacunas se han enfocado en fragmentos representativos de los genes rOmpA y rOmpB como vacunas de ADN. Las inmunizaciones han conferido cierto grado de protección a ratones vacunados, cuando las inmunizaciones por ADN fueron seguidas por revacunaciones con la proteína recombinante homóloga. La proliferación y secreción de interferón gamma pudieron ser detectados después de la estimulación in vitro de los linfocitos de estos animales con antígeno total de $\mathrm{R}$ conorii ${ }^{38}$. En el caso de las rickettsias del grupo de las fiebres manchadas, todo parece indicar que el blanco adecuado para generar una vacuna es la proteína rOmpA, ya que tiene un papel importante en la adhesión inicial de $\mathrm{R}$ rickettsii a las células hospederos y en algunos ensayos se ha observado que todos los anticuerpos monoclonales hacia rOmpA inhiben el proceso de adhesión de rickettsia hacia las células L-929 con valores de inhibición de hasta $90 \% 39$. En contraste, los anticuerpos monoclonales hacia $\mathrm{rOmpB} 0$ lipopolisacáridos no han bloqueado su unión. La participación de $\mathrm{rmpB}$ dentro del proceso de infección aún no ha sido del todo establecida.

Una opción para el desarrollo de vacunas eficaces es la identificación de secuencias antigénicas en la proteína rOmpA, comunes en todas las especies de rickettsias del grupo de las fiebres manchadas para la generación de una vacuna monovalente o la producción de una vacuna polivalente que contenga los principales epítopes especie específicos de esta proteína tan importante para la supervivencia de la bacteria.

Por último, la identificación de las pulgas de diversos mamíferos, como transmisores de algunas rickettsias, ha incrementado el impacto potencial de la enfermedad en la población humana, ya que su presencia es común en animales domésticos que cohabitan con el ser humano. Rolain y cols, en 2003 han presentado evidencias de que las pulgas de gato en Francia se encuentran comúnmente coinfectadas por $\mathrm{R}$ felis $\mathrm{y}$ algunas especies de Bartonella (incluyendo a B quintana ${ }^{40}$. El ciclo clásico de Rickettsia typhi involucra a las ratas y la pulga de la rata, Xenopsylla cheopis, como principal vector, pero también ha sido descrita su presencia en pulgas (Ctenocephalides felis) colectadas de zarigüeyas ${ }^{41}$.

En conclusión, el concepto de enfermedades emergentes o re-emengentes se ha aplicado a un sinnúmero de enfermedades que aquejan a nuestra población y presentan una característica en común: han existido métodos de diagnóstico adecuados que han permitido su detección durante varios años. Sin embargo, existen enfermedades que han pasado inadvertidas como es el caso de la rickettsiosis, de la cual se desconoce su magnitud y trascendencia en la población.

En la actualidad, los métodos de diagnóstico inmunológicos (IFI y Western blot) y moleculares (PCR-RFLPs) nos permiten tener una panorámica renovada de las enfermedades rickettsiales. Se pueden realizar tamizajes generales utilizando las pruebas serológicas para $\mathrm{OmpA}$ y $\mathrm{rOmpB}$, o por medio de PCR, de la lipoproteína de $17 \mathrm{kDa}$. La caracterización de las especies en el grupo tifo se puede realizar por medio del Western blot con la proteína rOmpB, y por medio de PCR-RFLPs de la proteína rOmpA en el caso de las rickettsias del grupo de las fiebres manchadas.

Las mismas proteínas membranales son las principales candidatas para la generación de vacunas de ADN. La búsqueda se ha concentrado en los epítopes compartidos por varias especies con el objeto de desarrollar una vacuna polivalente eficaz para el control y erradicación de las rickettsiosis. 


\section{REFERENCIAS}

1. La Scola B, Raoult D. Laboratory diagnosis of rickettsioses: Current approaches to diagnosis of old and new rickettsial diseases. J Clin Microbiol 1997; 35: 2715-27.

2. Tamura A, Ohashi $\mathrm{N}$, Urakami $\mathrm{H}$, Miyamura $\mathrm{S}$. Classification of Rickettsia tsutsugamushi in a new genus, Orientia gen. Nov., as Orientia tsutsugamushi comb. nov. Int J Syst Bacteriol 1995; 45: 589-91.

3. BARON S. Medical Microbiology, 4th Ed. University of Texas Medical Branch at Galveston, Galveston, TX 1996.

4. Beati L, Raoult D. Spotted fever group rickettsiae. En: Rickettsiae and rickettsial diseases. Kazar J y Toman R. Eds. Proceedings of the V the International Symposium, 1996, Slovak Academy of Sciences. Bratislava, 1996; 134-8.

5. McDade JE, Fishbein DB. Rickettsiaceae: The Rickettsiae. In: Laboratory diagnosis of infectious diseases, principles and practice. Vol II. Lennette $\mathrm{EH}$, Halonen $\mathrm{P}$ y Murphy FA, eds. SpringerVerlag, New York 1988.

6. Zavala-VelázQuez JE, Yu X-H, WalKer DH. Unrecognized spotted fever group rickettsiosis masquerading as dengue fever in México. Am J Trop Med Hyg 1996; 55: 157-9.

7. Zavala-Velázquez JE, Ruiz-Sosa JA, Sánchez-Elúas RA, Becerra-Carmona G, Walker DH. Rickettsia felis rickettsiosis in Yucatan. Lancet 2000; 356: 1079-80.

8. Zavala-Velázquez JE, Zavala-Castro JE, Vado-Solís I, Ruiz-SOSA JA, Morón CG, BOUYer DH ET AL. Identification of Ctenocephalides felis fleas as a host of Rickettsia felis, the agent of a spotted fever rickettsiosis in Yucatan, Mexico. Vector Borne Zoonotic Dis 2002; 2: 69-75.

9. Crocquet-Valdés PA, Weiss K, Walker DH. Sequence analysis of the $190 \mathrm{kDa}$ antigen-encoding gene of Rickettsia conorii (Malish 7 strain). Gene 1994; 140: $115-9$

10. Walker DH, Herrero-Herrero J, Ruiz-Beltrán R, Buшon-Sopelama A, Ramón-Hidalgo A. The pathology of fatal Mediterranean spotted fever. Am J Clin Pathol 1987; 87: 669-72.

11. Raoult D, Zuchew P, Weiluer PJ, Charrel C, San MarCo JL, GaLais H. Incidence, clinical observations, and risk in the severe form of Mediterranean spotted fever among patients admitted to hospital in Marseilles 1983-1984. J Infect 1986; 12: 111-6.

12. JERRELS TR. Mechanisms of immunity to Rickettsia species and Coxiella burnetti: En: Walker DH, Ed. Biology of rickettsial disease. CRC Pres Boca Raton, Fl. 1988; 79-100.

13. HerRero-Herrero Ji, WalKer DH, Ruiz-Beltrán R. Immunohistochemical evaluation of the cellular immune response to Rickettsia conorii in Taches noires. J Infect Dis 1987; 155: 802-5.

14. Kenyon RH, Pedersen CH. Immune response to Rickettsia akari infection in congenitally athimic nude mice. Infect Immun 1980; 28: 310-3.

15. Cimari E, Milano S, D’Agostino P, Arcoleo F, Stassi G, GaLuzo. Depression of CD4 $\mathrm{T}$ cell sub-sets and alteration in cytokine profile in boutonneuse fever. J Infect Dis 1996; 174: 1051-7.

16. IKeDa M, TAKaHashi $\mathrm{H}$, Yoshida S. HLA-DR+CD-+ and CD8+ cells are increased but CD4+CD45RA+ cells are reduced in the peripheral blood in human scrub typhus. Clin Immunol Immunopathol 1994; 72: 402-4.

17. DíaZ M, Feng H-M, WALKER DH. Rickettsia conorii antigen presentation to CD8 T-lymphocytes by a murine endothelial cell line. En: Rickettsiae and rickettsial diseases at the turn of the third millenium. Raoult D y Brouqui P, Eds. Elsevier, Paris, FR. 1999; 109-15.

18. Walker DH, Popov VL, Feng HM. Establishment of a novel endothelial target mouse model of a typhus group rickettsiosis: evidence for critical roles for gamma interferon and CD8 T lymphocytes. Lab Invest 2000; 80: 1361-72.

19. Gately MK, Renzetti LM, Magram J, Stern AS, Adorin L, GUBIER U. The interleukin-12/interleu-12-receptor system role in normal and pathologic immune response. Annu Rev Immunol 1998; 16: 495-521.

20. Feng H-M, Prov VL, Yuoh G, WaLker DH. Role of T-lymphocyte subsets in immunity to spotted fever group rickettsiae. J Immunol 1997; 158: 5314-20.

21. Feng H-M, WaLKER DH. Interferon-yyy and tumor necrosis factor-aaa exert their antirickettsial effect via induction of synthesis of nitric oxide. Am J Pathol 1993; 143: 1016-23.

22. Silverman DJ, SANTUCCI LA. Potential for free radical induced lipid peroxidation as a cause of endothelial cell injury in Rocky Mountain spotted fever. Infect Imunn 1988; 56: 3110-5. 
23. SPORN LA, MARDER VJ. Interleukin-1aaa production during Rickettsia rickettsii infection of cultured endothelial cells: potential role in autocrine cell stimulation. Infect Immun 1996; 64: 1609-13.

24. Crocquet-Valdés PA, Díaz-Montero CM, Feng H-M, L H, BARRET AD, WALKER DH. Immunization with a portion of rickettsial outer membrane protein A stimulates protective immunity against spotted fever rickettsiosis. Vaccine 2001; 20: 979-88.

25. FAN M-Y, YU XJ, WaLKER DH. Antigenic analysis oh Chinese strains of spotted fever group Rickettsiae by protein immunoblotting. Am J Trop Med Hyg 1988; 39: 497-501.

26. Walker DH, Feng HM, Saada Ji, Croquet-Valdés P, Radulovic S, Popov VL et al. Comparative antigenic analysis of spotted fever group rickettsia from Israel and other closely related organisms. Am J Trop Med Hyg 1995; 52: 569-76.

27. Anderson BE, McDonald GA, Jones DC, Regnery $\mathrm{RL}$. A protective antigen protein antigen of Rickettsia rickettsii has tandemly repeated nearidentical sequences. Infect Immun 1990; 58: 2760-9.

28. Gilmore RD JR. Comparison of the rOmpA gene repeat regions of Rickettsiae reveals speciesspecific arrangements of individual repeating units. Gene 1993; 125: 97-102.

29. Regnery RL, Spruil CL, Pukatys BD. Genotypic identification of Rickettsiae and estimation of intraespecies sequence divergence for portions of two rickettsial genes. J Bacteriology 1991; 173: 1576-89.

30. $\mathrm{Xu}$ W, Raoult D. Distribution of immunogenic epitopes on the two major immunodominant proteins (rOmpA and $\mathrm{rOmpB}$ ) of Rickettsia conorii among the other rickettsiae of the spotted fever group. Clin Diag Lab Immunol 1997; 4: 75363.

31. Roux V, Fournier P-E, Raoult D. Differentiation of spotted fever group Rickettsiae by sequencing and analysis of restriction fragment length polymorphism of PCR- amplified DNA of the gene encoding the protein rOmpA. J Clin Microbiol 1996; 34: 2058-65.
32. Bouyer DH, Stenos J, Crocouet-Valdés P, Morón CG, Popov VL, Zavala-VelázQuez JE et al. Rickettsia felis: molecular characterization of a new member of the spotted fever group. Int J Syst Evol Microb 2001; 51: 339-47.

33. ANDERSSON JO, ANDERSSON SGE. A century of typhus, lice and Rickettsia. Res Microbiol 2000; 151: 143-50.

34. ANDERSSon SGE, Zomorodipour A, Andersson JO, Sicheritz-Pontén T, Alsmark UC, Podowski RF et al. The genome sequence of Rickettsia prowazekii and the origin of mitochondria. Nature 1998; 396: 133-40.

35. Gilmore RD, Joste N, McDonald GA. Cloning, expression, and sequence analysis of the gene encoding the $120 \mathrm{kD}$ surface-exposed protein Rickettsia rickettsii. Mol Microbiol 1989; 3: 1579-86.

36. Ching WM, Wang H, Dasch GA. Identification and characterization of linear epitopes recognized by mouse monoclonal antibodies on the surface protein antigen of Rickettsia prowazekii. En: Rickettsiae and rickettsial diseases at the turn of the third millenium. Raoult D y Brouqui P, Eds. Elsevier, Paris, FR. 1999; 16-22.

37. ChIng WM, WANG H, DASCH GA. Identification and characterization of epitopes on the $120 \mathrm{kDa}$ surface protein antigen of Rickettsia prowazekii with rabbit antisera. Infect Immun 1996; 64: 1413-9.

38. Díaz-Montero CM, Feng HM, Crocouet-Valdés PA, WALKER DH. Identification of protective components of two major outer membrane proteins of spotted fever group Rickettsiae. Am J Trop Med Hyg 2001; 65: 371-8.

39. L H, WaLKER DH. rOmpA is a critical protein for the adhesion of Rickettsia rickettsii to host cells. Microb Pathog 1998; 24: 289-98.

40. Rolain J-M, Franc M, Davoust B, Raoult D. Molecular detection of Bartonella Quintana, B koehlarae, B henselae, B clarridgeiae, Rickettsia felis and Wolbachia pipientis in cat fleas, France. Emerg Infect Dis 2003; 9: 338-42.

41. Azad AF, Radulovic S, Higgins Ja, Noden BH, TROYER JM. Flea-borne rickettsiosis: ecologic considerations. Emerg Infect Dis 1997; 3: 319-27. 